\title{
Effect of Postural Changes on Complexity Measures of Heart Rhythm in Late Adolescents
}

\author{
Tomasz H Wierzba, Hanna Cwikalowska-Grudzinska, Piotr Badtke, Stanislaw Zajaczkowski \\ ${ }^{1}$ Department of Physiology, Medical University of Gdansk, Gdansk, Poland
}

\begin{abstract}
Even trivial changing of body position from supine to sitting triggers complex cardiovascular adaptive response to prevent brain ischemia.

The study was performed to investigate heart rate asymmetry (HRA) and entropy-based heart rate variability (HRV) in stationary conditions in two body positions.

Forty male students, aged 18-20 participated in this study. High resolution (4 kHz) ECG was recorded for 15 minutes; first in supine and after that in sitting position. 512 beat-to-beat interval (RRi) time-series were selected from each recording. Entropy-based parameters such as sample entropy (SampEn) and approximate entropy (ApEn) were computed. Heart cycles shorter (acceleration) or longer (deceleration) from the previous ones and sequential monotonic changes of RRi were also discriminated. Number of HR sequential runs significantly correlated with SampEn and ApEn both in sitting and supine position. Postural change from supine to sitting position resulted in a significant HR increase from 69 to 77 bpm. Both SampEn and ApEn were decreased after changing position (from 1.54 to 1.20 and from 1.23 to 1.08, respectively).

Our data obtained from nonlinear HRV analysis indicate a reduction of cardiac control complexity following postural upright changing of body position.
\end{abstract}

\section{Introduction}

Each person deals with orthostatic stresses every day. As a result of changing posture from supine to sitting, a volume of blood moves from chest into lower parts of the body. Cardiovascular adaptive mechanisms and reflexes are responsible for heart rate (HR) and blood pressure (BP) adjustments. Autonomic nervous system (ANS) plays major role in maintaining cardiovascular homeostasis. ANS divides in two functionally different parts (sympathetic and parasympathetic) that play in concert to control HR and BP. In our work we investigated beat to beat changes of HR as they reflect ANS regulatory control on the heart. Since temporal changes of HR are mostly distributed in a non-gaussian pattern, our study was focused on heart rate asymmetry (HRA) [1] and non-linear parameters of heart rate variability (HRV) [2, 3].
The study was performed to compare the heart rhythm complexity in stationary conditions in two body positions with use of HRA analysis and entropy-based HRV measures.

\section{Materials and Methods}

The study was conducted in accordance with the Declaration of Helsinki and the protocol was approved by the Independent Bioethics Commission.

Forty male students aged 18-20 were enrolled to the study. Prior to the ECG recording procedure volunteers remained possibly quiescent and relaxed in supine position for at least 10 minutes. Following the adaptation to the experimental conditions 4-kHz ECG (LabChart 8 Pro software, AdInstruments, Sydney, Australia) was recorded for 15 minutes first in supine and after that in sitting position. R waves were identified and RR intervals (RRi) were discriminated with LabChart 8 pro software using standard settings. After correction of ectopic and artifactual peaks, 512 beat-to-beat intervals (RRi) were selected from each recording. Entropy-based parameters such as approximate entropy (ApEn) and sample entropy (SampEn) were computed with use of Kubios 3.2 HRV Pro software (Kuopio, Finland). Heart cycles shorter (acceleration) or longer (deceleration) from the previous ones and sequential monotonic (acceleration or deceleration runs) changes of RRi were discriminated with use of dedicated software (Microsoft Excel, USA).

Statistical analysis was performed using GraphPad Prism 7 (La Jolla, CA, USA) and Statistica 13 software (StatSoft, Tulsa, USA). All data sets were tested for normality with Shapiro-Wilk test. Statistical analysis was based on Wilcoxon and paired Student t-tests, depending whether data had normal or non-normal distribution. Linear regression analysis between selected pairs variables was performed and Pearson correlation coefficient $r$ was calculated. All data are shown as mean values \pm standard deviation of the mean $( \pm S D)$. P values $<0.05$ were considered statistically significant. 


\section{Results}

Postural change from supine to sitting position resulted in a significant HR increase from $69.2 \pm 9.3$ to $77.1 \pm 10.2$ beats per minute (bpm; $\mathrm{p}<0.01$ ), and an increase of RRi from 780 to 881 ms. As shown in Figure 1, both ApEn (A) and SampEn (B) decreased after changing position from supine to sitting: from 1.23 to 1.08 and from 1.54 to 1.20 $(\mathrm{p}<0.05)$ respectively.
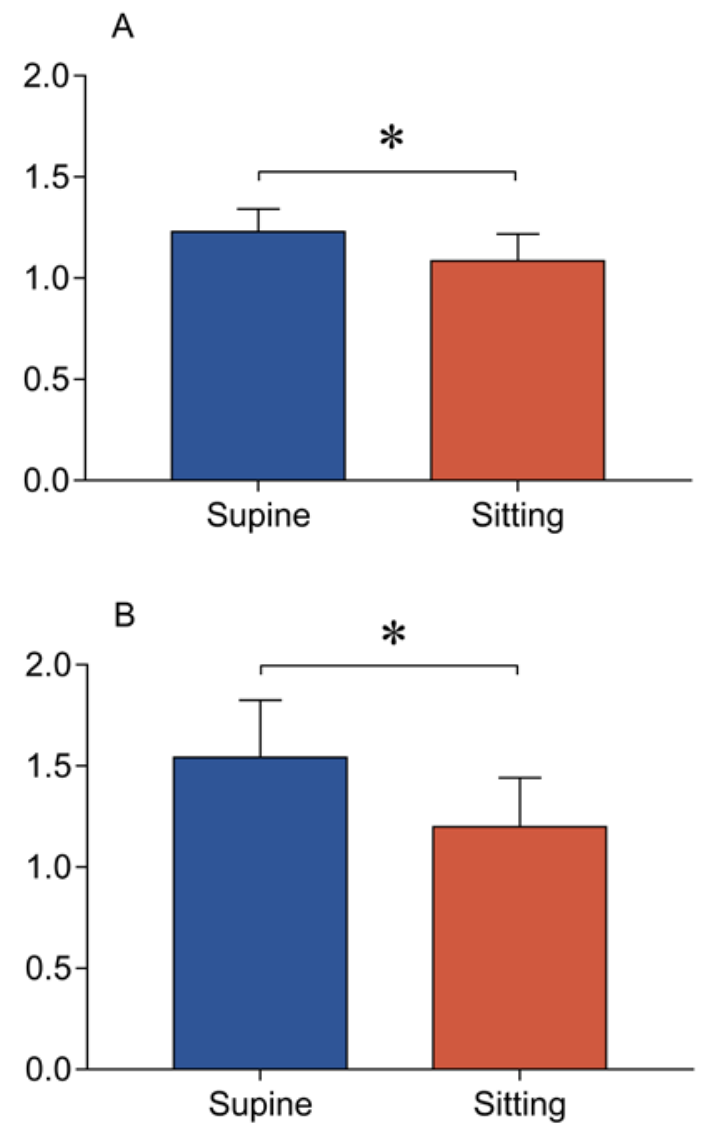

Figure 1. Non-linear HRV parameters: Approximate Entropy (A) and Sample Entropy (B) after changing position from supine to sitting. * $-\mathrm{p}<0.05$.

The ratio of decelerations to accelerations of HR cycles was higher in supine compared to sitting position, $(0.93 \pm 0.20$ to $0.90 \pm 0.18$;-p $<0.05$; Figure 2$)$. The total number of monotonic sequential RRi changes was significantly lower $(-18.5 \%)$ in sitting than in supine position (Figure 3). The observed difference was mainly covered by a decrease runs of lengths of 2 heart beats ($52 \%)$. In contrast to the short monotonic RRi sequences consisting of 2 to 3 subsequent heart cycles, the number of longer monotonic runs (lengths of 4 to 9 heart beats) was significantly higher in sitting position (+263\%).

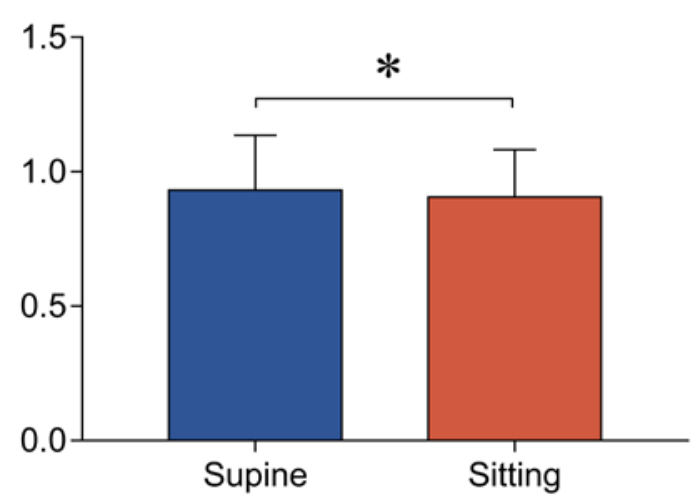

Figure 2. Deceleration to acceleration ratio of HR cycles, $*-\mathrm{p}<0.05$.

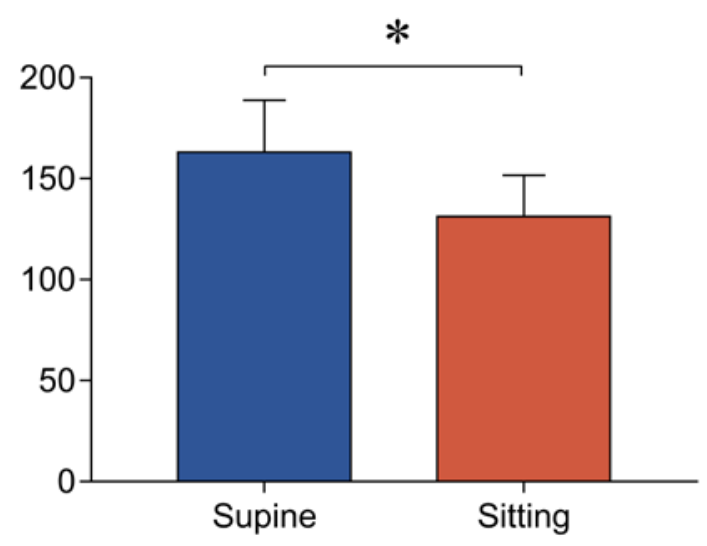

Figure 3. Total number of monotonic sequential RRi changes. * $\mathrm{p}<0.05$

Regardless the body position, the total number of HR sequential runs significantly correlated with ApEn: $r=0.7093$ (supine; Figure $4 \mathrm{~A}$ ) and $r=0.7076$ (sitting; Figure 4 B). Similar correlation was observed with SampEn: $r=0.6597$ (supine; Figure $4 \mathrm{C}$ ) and $r=0.7076$ (sitting; Figure $4 \mathrm{D}$ ). The ratio of decelerations to accelerations did not significantly correlate with either ApEn: $r=0.4800$ (supine; Figure $4 \mathrm{E}$ ) and $r=0.3638$ (sitting; Figure $4 \mathrm{~F}$ ) or SampEn: $r=0.4136$ (supine; Figure $4 \mathrm{G}$ ) and $r=0.3589$ (sitting; Figure $4 \mathrm{H}$ ). Changes in deceleration to acceleration ratio evoked by the postural changes were closely related to both entropy HRV parameters: ApEn $(r=0.6298)$ and SampEn $(r=0.5809$; Figure $5 \mathrm{~A}$ and $\mathrm{B})$. 
A

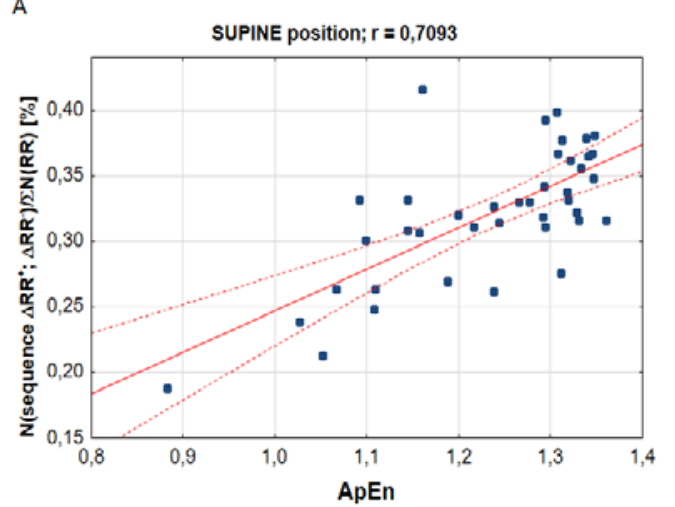

B
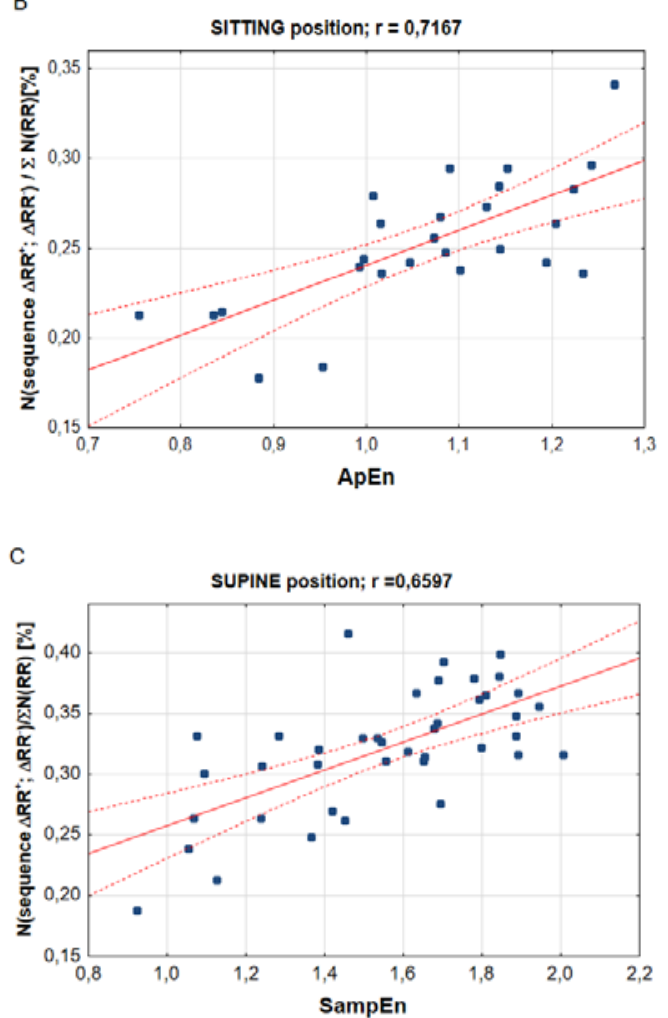

D

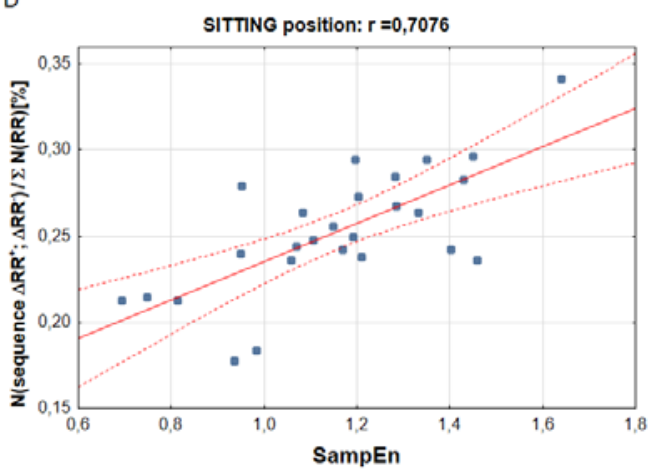

E

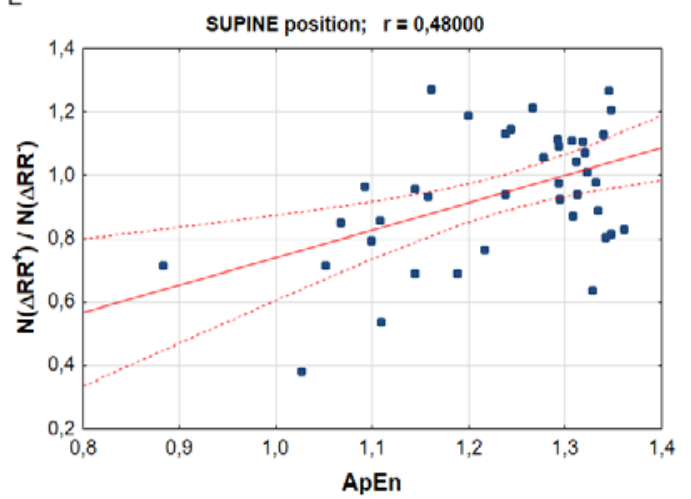

F

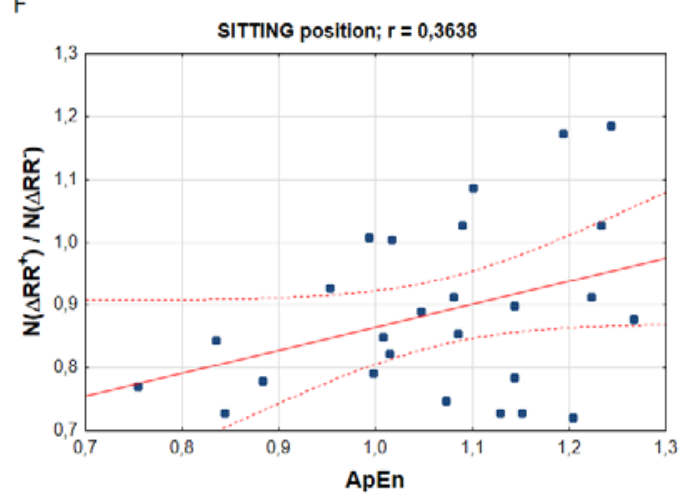

G

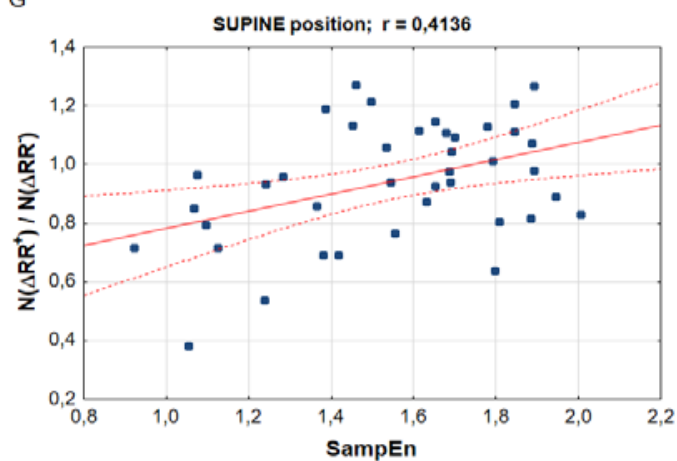

$\mathrm{H}$

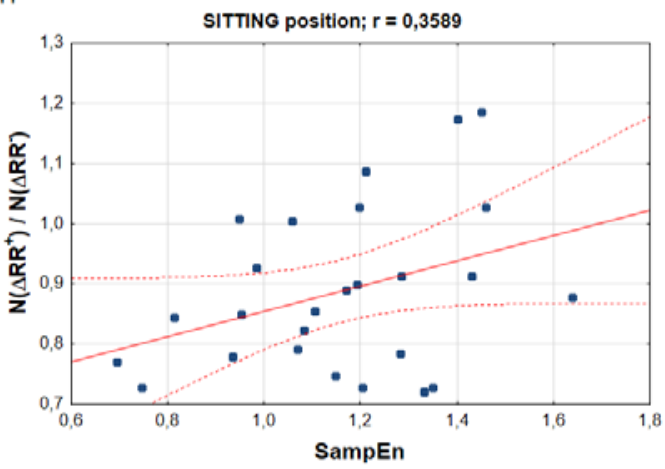

Figure 4. Correlation between entropy measures (ApEn and SampEn) and HRA parameters. Total number of sequential runs vs. ApEn supine (A) and sitting (B) vs. SampEn supine (C) and sitting (D); the ratio of decelerations to accelerations vs ApEn supine (E) and sitting (F) vs SampEn supine $(\mathrm{G})$ and sitting $(\mathrm{H})$. Abbreviations are explained in text. 
A

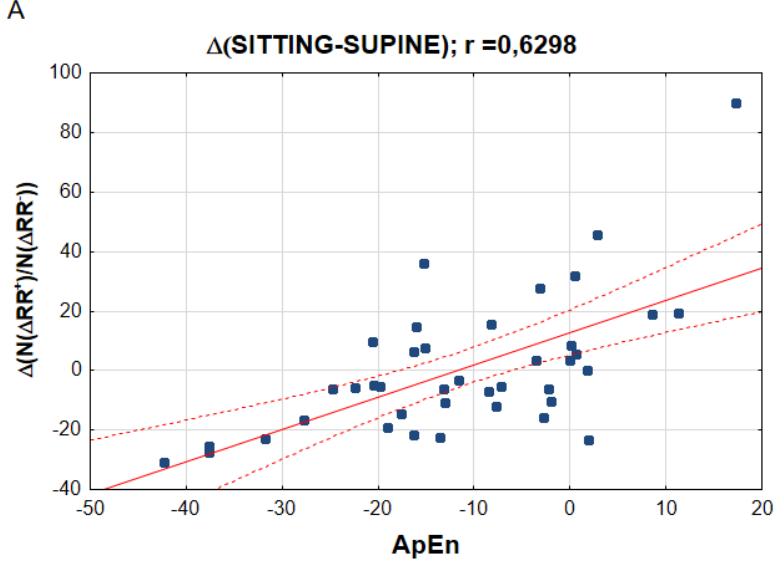

B

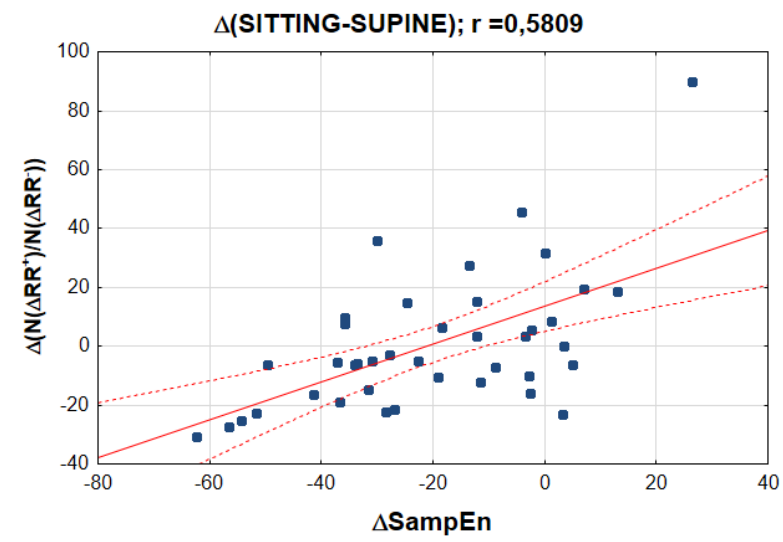

Figure 5. Changes in deceleration to acceleration ratio evoked by the postural changes. A - ApEn, B - SampEn.

\section{Discussion}

As a result of a postural changes from supine to sitting position, a person is exposed to orthostatic stress. As confirmed by the significant increase in HR the functional effects of such a relatively small postural challenge are not negligible. Our data confirmed the value of the beat-to-beat analysis based on nonlinear dynamics as suggested much earlier by Richman and Moorman [4].

The main response to changing position form supine to sitting was an increase in HR with a decrease in both ApEn and SampEn. This suggests that routine change of position affects complexity and regularity of autonomic regulation upon the heart. Lower value of ApEn in sitting position indicates higher degree of regularity in HR. Consistently, lower value of SampEn reflects more self-similarity of RRi series in sitting position. Our data might suggest that during orthostatic stress ANS control upon the heart is determined by fewer deterministic regulators of functional relevance. As seen by a significant HR increase, the sympathovagal balance was apparently shifted towards the sympathetic prevalence. A decreased number of the shortterm monotonic changes of RRi intervals accompanied by a substantial increase of the longer monotonic RRi sequences suggests simultaneous vagal withdrawal and sympathetic activation.

\section{Summary}

HRA corresponds to the entropy based HRV measures. Nonlinear mechanisms related to complexity of the heart rate control systems are involved in cardiac responses to changes in body position.

\section{Acknowledgments}

The study was supported by Medical University of Gdansk ST: 02-0036/07/261

\section{References}

[1] J. Piskorski and P. Guzik, "The structure of heart rate asymmetry: deceleration and acceleration runs," Physiol. Meas., vol. 32, no. 8, p. 1011, 2011.

[2] H. Young and D. Benton, "We should be using nonlinear indices when relating heart-rate dynamics to cognition and mood," Sci Rep, vol. 5, p. 16619, 2015.

[3] C. Fiskum et al., "Non-linear heart rate variability as a discriminator of internalizing psychopathology and negative affect in children with internalizing problems and healthy controls," Front Physiol, vol. 9, p. 561, 2018.

[4] J. S. Richman and J. R. Moorman, "Physiological time-series analysis using approximate entropy and sample entropy,” Am J Physiol Heart Circ Physiol, vol. 278, no. 6, H2039-49, 2000.

Corresponding address:

Tomasz H. Wierzba,MD, PhD

Medical University of Gdansk

Department of Physiology

1 Debinki Street

80-211 Gdansk, Poland

twierzba@gumed.edu.pl 